\title{
Anti-Müllerian Hormone Accelerates Pathological Process of Insulin Resistance in Polycystic Ovary Syndrome Patients
}

Authors

Xiang-Juan Li ${ }^{1}{ }^{*}$, Hui Wang ${ }^{2}$, Dan-Yang Lu ${ }^{3}$, Tian-Tian $\mathrm{Yu}^{3}$, Kamran Ullah", Xin-Yan Shi ${ }^{1}$, Yong-Hai Shen ${ }^{1}$, Xiao-Yang Fei ${ }^{1}$, Zhen-Yun Lin ${ }^{1}$, He-Feng Huang ${ }^{3,5}$, Xian-Hua Lin ${ }^{5}$

Affiliations

1 Department of Obstetrics and Gynecology, Hangzhou Women's Hospital, Hangzhou, China

2 Department of Obstetrics and Gynecology, Maternal and Child Health Hospital of Songjiang District, Shanghai, China

3 Obstetrics and Gynecology Hospital of Fudan University, Shanghai, China

4 Department of Biological Sciences (Zoology), University of Lakki Marwat, Khyber Pakhtunkhwa, Pakistan

5 The Key Laboratory of Reproductive Genetics, Ministry of Education (Zhejiang University), Hangzhou, China.

Key words

anti-Mullerian hormone, insulin resistance, apoptosis, pancreatic islet cells

received $\quad 05.02 .2021$

accepted after revision $\quad 26.04 .2021$

Bibliography

Horm Metab Res 2021; 53: 504-511

DOI 10.1055/a-1499-7718

ISSN 0018-5043

(c) 2021. Thieme. All rights reserved.

Georg Thieme Verlag KG, Rüdigerstraße 14,

70469 Stuttgart, Germany

Correspondence

Xian-Hua Lin

Obstetrics and Gynecology Hospital of Fudan University

419 Fangxie Road

Shanghai

China

Tel.: + 862133189900 , Fax: + 862164070434

xl_1290@126.com
He-Feng Huang

Obstetrics and Gynecology Hospital of Fudan University

419 Fangxie Road

Shanghai

China

Tel.: + 862133189900 , Fax: + 862164070434

A Supplementary material is available under

https://doi.org/10.1055/a-1499-7718

\section{ABSTRACT}

Insulin resistance (IR) is one of the most common features of polycystic ovary syndrome (PCOS), which is related to obesity. Whether increased anti-Müllerian hormone (AMH) levels in PCOS are involved in the pathogenesis of insulin resistance remains unclear. We investigated serum levels of leptin and $\mathrm{AMH}$ along with basic clinical and metabolic parameters in 114 PCOS patients and 181 non-PCOS women. PCOS patients presented higher fasting blood glucose, insulin concentrations and Homeostatic Model Assessment of Insulin Resistance (HOMA-IR) in addition to body mass index (BMI), lipids profiles and hormone levels. HOMA-IR showed a positive correlation with BMI, $\mathrm{AMH}$, leptin, and low-density lipoprotein-cholesterol (LDL-C) levels. Interestingly, AMH is strongly positively correlated with HOMA-IR and insulin concentrations for 1 st and 2 nd hours of glucose treatment after fasting. Among PCOS women with $B M I \geq 25 \mathrm{~kg} / \mathrm{m}^{2}$, high AMH level group showed an increased HOMA-IR when compared to normal AMH level. However, among PCOS women with normal BMI, women with high AMH presented an elevated fasting insulin levels but not HOMA-IR when compared to normal AMH group. In vitro treatment of isolated islet cells with high concentration of leptin $(200 \mathrm{ng} /$ $\mathrm{ml}$ ) or high leptin plus high concentration of AMH $(1 \mathrm{ng} / \mathrm{ml})$ significantly enhanced insulin secretion. Importantly, co-treatment of AMH plus leptin upregulates the expression of pro-apoptotic proteins, such as Bax, caspase-3, and caspase- 8 after incubating with a high level of glucose. These results suggest that $\mathrm{AMH}$ may involve in the pathological process of pancreatic $\beta$-cells in obese PCOS women.

These two authors contributed equally to this study 


\section{Introduction}

Polycystic ovary syndrome (PCOS) is the most common endocrine disorder in reproductive-age women, with a prevalence of $15 \%$ depending on the diagnostic criteria used and the population studied [1]. Its clinical manifestations may include menstrual irregularities, signs of hyperandrogenism, and obesity. Insulin resistance (IR) is one of the most common features of PCOS and is associated with an increased risk of type 2 diabetes and cardiovascular events, with a consequent negative effect on long-term health [2]. Although IR is not required for a diagnosis of PCOS, it is clear that intrinsic IR is present in many women with PCOS [3]. However, the underlying etiology and pathogenesis of PCOS remain poorly understood, and there is no effective method of preventing the disease.

As a member of the transforming growth factor- $\beta$ (TGF- $\beta$ ) family, anti-Müllerian hormone (AMH) is expressed by granulosa cells (GCs) of early developing follicles in the ovary during the reproductive age $[4,5]$. AMH is considered a reliable of ovarian reserve in humans and strongly correlate with the number of antral follicles $[6,7]$. In the ovary, the main physiologic role of AMH seems to be limited to the inhibition of the early stages of follicular development [8] and preventing the recruitment of non-dominant follicles [9]. Although AMH is mainly thought to play a local role in ovarian follicle recruitment and development [7], it is also available in the peripheral circulation.

IR results from impaired insulin signaling in target tissues that leads to increased levels of insulin required to control plasma glucose levels. IR and pancreatic $\beta$-cell dysfunction are two major pathophysiological characteristics of type 2 diabetes mellitus $\left(\mathrm{T}_{2} \mathrm{DM}\right)$, thus pancreatic $\beta$-cells play a crucial role in $\mathrm{T}_{2} \mathrm{DM}$ pathogenesis $[10,11]$. In the early stage of $\mathrm{T}_{2} \mathrm{DM}$ development, the pancreas first increases insulin release by improving the function of the $\beta$-cells or increasing their number when IR occurs in peripheral tissues. The $\beta$-cell apoptosis caused by high-glucose toxicity results in decreased $\beta$-cell function and insulin sensitivity, further reducing insulin secretion in diabetic patients with long-term hyperglycemia.

It is well known that obesity is always related to IR in women with PCOS, while whether the increased AMH levels in PCOS is involved in the pathological process of IR in reproductive-age women with PCOS and whether the increased AMH has an effect on pancreatic $\beta$-cells still remains unclear. Hence, the aim of the present study was to investigate the underlying relationship between serum AMH levels and the HOMA-IR, and also with the insulin levels after glucose stimulation. Furthermore, we conducted an in vitro model to examine the effects of high $\mathrm{AMH}$ in association with high leptin levels on insulin secretion and the expression of pancreatic $\beta$-cell pro-apoptosic genes in the presence of high glucose concentrations.

\section{Subjects and Methods}

\section{Samples collection}

The study was approved by the ethical committee of School of Medicine, Zhejiang University, Hangzhou, China. Total of 345 women were recruited from the Reproductive Center of Hangzhou Women's Hospital between 2013 and 2015. Fifty women whose age was more than 37 years and/or with hypertension and other medical diseases were excluded, 295 women including 114 women with PCOS and 181 women without PCOS were enrolled. After stratified by BMI, PCOS women were subgrouped into normal BMI (18.5-24.9 $\mathrm{kg} / \mathrm{m}^{2}, \mathrm{n}=73$ ) and high BMI groups ( $\left.\geq 25 \mathrm{~kg} / \mathrm{m}^{2}, \mathrm{n}=41\right)$. Among PCOS women with normal BMI, 35 women with normal AMH level and 38 women with high AMH level were analyzed. And among obese PCOS women, 22 women with normal AMH and 19 women with high AMH level were analyzed. The diagnosis of PCOS was made according to the Rotterdam Consensus [12]. Women with hyperprolactinemia, hypothyroidism, androgen-secreting tumors, Cushing syndrome, congenital adrenal hyperplasia, or diabetes were also excluded.

\section{Analysis of serum hormone and biochemical indicators analysis}

Basal hormone and metabolic profiles of patients were examined on days 2-5 of menstruation after fasting for 10-12 hours, in subjects who did not take any medications, including hormones, in the past 3 months. The concentrations of basal sex hormone and insulin levels were measured at the clinical chemistry laboratory of Hangzhou Women's Hospital (DX8001; Beckman Instrument). Serum glucose levels were determined using an automated hexokinase method (AU5821; Beckman Instrument), the triglyceride contents were measured by a standard colorimetric assay (AU5821; Beckman Instrument) and the cholesterol levels were measured by enzyme assays (AU5821; Beckman Instrument). The amount of $\mathrm{AMH}$ was quantified with automated chemiluminescent immunoassay method (Roche Diagnostics; Basel, Switzerland), measuring a range of $0.01-23 \mathrm{ng} / \mathrm{ml}$, the detection sensitivity of $0.3 \mathrm{ng} / \mathrm{ml}$ and intra- and inter-assay coefficients of variation < $10 \%$. HOMA-IR index was calculated according to the formula: HOMA-IR = (fasting glucose) $(\mathrm{mmol} / \mathrm{l}) \times($ fasting insulin $)(\mu \mathrm{U} / \mathrm{ml}) / 22.5)$ [13]

\section{Pancreatic islet isolation and insulin secretion}

In anesthetized 8-week-old rats, $2 \mathrm{mg} / \mathrm{ml}$ collagenase (Worthington Biochemical, Lakewood, NJ) was injected into the bile duct as previously described $[14,15]$. The pancreas was isolated, with static incubation at $38^{\circ} \mathrm{C}$ for 20 minutes followed by shaking incubation at $38^{\circ} \mathrm{C}$ for 7 minutes. After ficoll gradient separation, freshly isolated islets of similar size were handpicked under a stereomicroscope. The islets (20 per well) were preincubated in RPMI-1640 (Sigma-Aldrich, St. Louis, MO, USA) washed, and incubated in $500 \mu \mathrm{l}$ fresh RPMI-1640 containing $11.1 \mathrm{mmol} / \mathrm{l}$ glucose, supplemented with $10 \%$ fetal calf serum that contained high glucose $(25 \mathrm{mmol} / \mathrm{l})$ and this was incubated with different concentration of recombinant rat leptin (L5073, Sigma-Aldrich) and/or r-AMH (CSBEP001666RA, CUSABIO) for 30 minutes $\left(37^{\circ} \mathrm{C}\right)$. Fifty microliters of the medium were removed for insulin analysis and the islets were extracted and stored $\left(-80^{\circ} \mathrm{C}\right)$ for protein assay and western blotting. After incubation for 24 hours, cells were treated with different concentration of leptin ( 0 , Tris- $\mathrm{HCl}, 25,50,100,200 \mathrm{ng} / \mathrm{ml}$ ).

\section{Western blotting}

Western blotting was performed as described previously [16]. The islets samples were lysed and centrifuged before measuring the concentration of protein. In brief, the extracted protein was trans- 
ferred to polyvinylidene difluoride membranes and incubated overnight at $4{ }^{\circ} \mathrm{C}$ with rabbit polyclonal anti- $\alpha$-caspase-3 (1:1000; Cell Signaling Technology, Boston, CA, USA), anti- $\alpha$-caspase-8 (1:1000; Cell Signaling Technology, Boston, CA, USA), anti- $\alpha$-caspase- 9 (1:1000; Cell Signaling Technology, Boston, CA, USA), anti-Bax (1:1000; Abcam, Cambridge, MA, USA) and human polyclonal anti- $\beta$-actin (1:10 000; Abcam). The samples were then incubated with fluorescence-labeled anti-mouse IgG or anti-rabbit IgG antibody (1:5000, Dylight 680 or $800, \mathrm{KPL})$ for $1 \mathrm{~h}$ at room temperature, and, the protein bands were obtained with enhanced chemiluminescence (Millipore). Image of WB was captured by a CCD camera and analyzed with an Odyssey Imager (Li-cor; Odyssey, NE, USA). By using this software, the intensity of the bands was translated into a numerical value - the brighter the band, the higher the number. The results of western blots were assessed visually by making comparisons between bands in different lanes.

\section{Statistical analyses}

All data are presented as mean \pm SD or SEM. An independent-sample $t$-test, the nonparametric test was used to evaluate the statistical significance between two groups. Associations between parameters were assessed using the Pearson correlation coefficient. Multiple linear regression analysis, using HOMR-IR, fasting insulin and insulin levels after oral administration of glucose as dependent variables, and, BMI, serum leptin levels, AMH levels, basal hormone levels, and lipids levels as independent variables, were calculated. SPSS (version 19.0 for Windows) was used for the statistical analysis. $p$-Values of $<0.05$ were considered to be statistically significant.

\section{Results}

\section{PCOS exhibit hyperinsulinemia and IR}

The flow chart of our prospective cohort study was presented in Fig. S1. Among 345 women of reproductive age, despite 50 women with advanced age and/or diabetes, hypertension or other medical diseases, 295 women (114 with PCOS and 181 healthy women) were recruited $(87.41 \%)$. Women's age was comparable. Serum metabolic parameters in women with or without PCOS were measured. There were no significant differences in height, basal FSH, $\mathrm{PRL}$, and serum triglyceride (TG) in both groups. However, body weight, body mass index (BMI), serum $\mathrm{LH}, \mathrm{E}_{2}$, total testosterone (TT), Total cholesterol (TC), LDL-c levels were higher and HDL-C was lower in PCOS group ( $\triangleright$ Table 1). Furthermore, levels of serum leptin $\mathrm{AMH}$, insulin, fasting glucose, and HOMA-IR were significantly higher in women with PCOS.

\section{Possible correlations between insulin resistance, insulin levels after glucose stimulation, and hormone and lipids parameters in PCOS women}

To investigate the risk factors for IR and insulin secretion after glucose stimulation in PCOS patients, we analyzed the correlation coefficients between HOMA-IR, and metabolic and hormone parameters. HOMA-IR was positively correlated to BMI, leptin, $\mathrm{AMH}$, and LDL-c levels ( $\triangleright$ Table 2). Glucose treatment for 1 st and 2 nd hours of fasting markedly increased BMI, insulin and leptin levels. AMH
- Table 1 Serum metabolic parameters between women with and without PCOS.

\begin{tabular}{|l|c|c|c|}
\hline & Non-PCOS & PCOS & p-Value \\
\hline No. of subjects & 181 & 114 & \\
\hline Age (years) & $27.71 \pm 4.17$ & $27.53 \pm 4.14$ & 0.372 \\
\hline Height $(\mathrm{cm})$ & $157.80 \pm 4.79$ & $159.98 \pm 4.96$ & 0.095 \\
\hline Weight $(\mathrm{kg})$ & $54.61 \pm 6.37$ & $62.16 \pm 12.45$ & $<0.0001$ \\
\hline BMI $\left(\mathrm{kg} / \mathrm{m}^{2}\right)$ & $21.22 \pm 2.32$ & $23.82 \pm 4.65$ & $<0.0001$ \\
\hline Basal LH level $(\mathrm{mlU} / \mathrm{ml})$ & $5.81 \pm 3.89$ & $10.95 \pm 6.65$ & $<0.0001$ \\
\hline Basal FSH level $(\mathrm{mlU} / \mathrm{ml})$ & $5.84 \pm 1.84$ & $5.93 \pm 1.46$ & 0.380 \\
\hline Basal E2 level $(\mathrm{pmol} / \mathrm{l})$ & $82.63 \pm 70.28$ & $176.55 \pm 90.66$ & $<0.0001$ \\
\hline TT level (nmol/l) & $0.63 \pm 0.44$ & $2.21 \pm 0.91$ & $<0.0001$ \\
\hline Basal PRL level $(\mathrm{ng} / \mathrm{ml})$ & $13.54 \pm 10.14$ & $15.21 \pm 8.64$ & 0.143 \\
\hline Serum triglyceride $(\mathrm{mM})$ & $1.52 \pm 1.39$ & $1.77 \pm 1.23$ & 0.079 \\
\hline Serum Cholesterol $(\mathrm{mM})$ & $4.73 \pm 0.81$ & $4.98 \pm 1.08$ & 0.022 \\
\hline Serum HDL-c $(\mathrm{mM})$ & $1.58 \pm 0.39$ & $1.43 \pm 0.45$ & 0.004 \\
\hline Serum LDL-c $(\mathrm{mM})$ & $2.55 \pm 0.63$ & $2.97 \pm 0.90$ & $<0.0001$ \\
\hline Serum leptin level $(\mathrm{ng} / \mathrm{ml})$ & $5.42 \pm 5.31^{\mathrm{a}}$ & $11.84 \pm 11.28 \mathrm{~b}$ & $<0.0001$ \\
\hline Serum AMH level $(\mathrm{ng} / \mathrm{ml})$ & $3.60 \pm 1.71$ & $7.66 \pm 6.48$ & $<0.0001$ \\
\hline Fasting glucose $(\mathrm{mM})$ & $4.46 \pm 0.59$ & $4.81 \pm 0.84$ & $<0.0001$ \\
\hline Fasting insulin $(\mu \mathrm{m} / \mathrm{ml})$ & $6.10 \pm 3.77$ & $15.17 \pm 8.84$ & $<0.0001$ \\
\hline HOMA-IR & $1.21 \pm 0.77$ & $2.63 \pm 1.50$ & $<0.0001$ \\
\hline & & & \\
\hline
\end{tabular}

Data $=$ mean \pm SD, $p$-values were calculated by $t$-test. BMI: Body mass index; LH: Luteinizing hormone; FSH: Follicle-stimulating hormone; E2: Estradiol; TT: Total testosterone; PRL: Prolactin; HDL-c: High-density lipoprotein-cholesterol; LDL-c: Low-density lipoprotein-cholesterol; HOMA-IR: Homeostatic Model Assessment for Insulin Resistance. ${ }^{a} n=47$, $\mathrm{b}_{\mathrm{n}}=91$.

showed a significant positive trend in correlation with HOMA-IR and insulin concentrations ( $\triangleright$ Table 2 ). Our present data suggest that high BMI and leptin levels were the main risk factor of IR, while the elevated $\mathrm{AMH}$ levels were associated with insulin secretion on the background of glucose stimulation.

In order to determine the interaction between obesity and elevated $\mathrm{AMH}$ levels on insulin resistance, we further analyzed the metabolic and hormone parameters among PCOS women after stratified by BMI (Fig. S1 and $>$ Table 3). No statistic correlation between leptin and AMH levels was found (data not shown). Surprising, PCOS women in high AMH level group showed an increased HOMA-IR when compared to normal AMH level group among obese PCOS women $(3.96 \pm 1.27$ vs. $3.02 \pm 1.42)$. The HOMA-IR in obese PCOS women with high AMH was the highest among the four subgroups ( $\triangleright$ Table 3). However, among PCOS women with normal BMI, women with high AMH presented an obviously elevated fasting insulin levels when compared to normal $\mathrm{AMH}$ group (20.14 \pm 11.96 vs. $10.71 \pm 4.98)$. But there was no difference in $\mathrm{HO}$ MA-IR between normal and high AMH groups among PCOS women with normal BMI ( $\triangleright$ Table 3). 
- Table 2 Correlation coefficients between insulin resistance, insulin levels after glucose stimulation, and hormone and lipids parameters in PCOS women.

\begin{tabular}{|c|c|c|c|c|}
\hline Parameters & $\begin{array}{l}\text { HO- } \\
\text { MA-IR } \\
(n=74)\end{array}$ & $\begin{array}{l}\text { Fasting } \\
\text { insulin } \\
\text { ( } \mathrm{n}=74 \text {, } \\
\text { mmol/I) }\end{array}$ & $\begin{array}{l}1 \text { hour } \\
\text { insulin } \\
(n=42 \text {, } \\
\mu \mathrm{U} / \mathrm{ml})\end{array}$ & $\begin{array}{l}2 \text { hours } \\
\text { insulin } \\
(n=42, \\
\mu \mathrm{U} / \mathrm{ml})\end{array}$ \\
\hline BMI $\left(\mathrm{kg} / \mathrm{m}^{2}\right)$ & $0.227^{\#}$ & 0.157 & $0.341^{\#}$ & $0.326^{\#}$ \\
\hline Leptin levels (ng/ml) & $0.326^{*}$ & 0.192 & $0.315^{\#}$ & 0.239 \\
\hline AMH levels (ng/ml) & $0.222^{\#}$ & $0.211^{\#}$ & $0.506^{\dagger}$ & 0.468 \\
\hline Basal LH level (mlU/ml) & 0.107 & -0.127 & 0.082 & $-0.321^{\#}$ \\
\hline Basal FSH level (mlU/ml) & 0.014 & 0.010 & $0.287^{\#}$ & 0.150 \\
\hline $\mathrm{TT}(\mathrm{nmol} / \mathrm{l})$ & -0.058 & 0.094 & 0.060 & 0.217 \\
\hline Serum TG (mmol/l) & 0.069 & 0.102 & -0.039 & -0.106 \\
\hline Serum TC (mmol/l) & 0.048 & -0.001 & 0.173 & 0.234 \\
\hline Serum HDL (mmol/l) & $-0.242^{\#}$ & $-0.284^{\#}$ & -0.161 & $-0.335^{\#}$ \\
\hline Serum LDL (mmol/l) & $0.248^{\#}$ & 0.139 & 0.133 & $0.287^{\#}$ \\
\hline
\end{tabular}

HDL: High-density lipoprotein; HOMA: Homeostatic Model Assessment; and LDL: Low-density lipoprotein. ${ }^{\dagger} p<0.001 ;{ }^{*} p<0.01$; $\# \mathrm{p}<0.05$.

\section{Co-treatment of high levels of AMH plus leptin increases insulin secretion in vitro}

Insulin itself appears to be a proximate and important quantitative contributor to insulin resistance [17]. In order to verify the direct effect of AMH on insulin secretion, isolated pancreatic (identified by incubating with dithizone, - Fig. 1a) were cultured for 2 hours in a high glucose medium (25 mmol/l) and treated with different $\mathrm{AMH}(0.25 \mathrm{ng} / \mathrm{ml}$ and $1 \mathrm{ng} / \mathrm{ml})$ concentrations, or with AMH $(1 \mathrm{ng} /$ $\mathrm{ml})$ plus leptin $(200 \mathrm{ng} / \mathrm{ml})$ concentrations. We found a significant increase in levels of insulin secretion in the group treated with high levels of AMH plus leptin, compared to the group treated with $\mathrm{AMH}$ only ( $\triangleright$ Fig. 1b). The results indicate that a slight increase in $\mathrm{AMH}$ concentrations may accelerate pancreatic islets insulin secretion.

\section{High AMH upregulated the expression of pro- apoptotic proteins in pancreatic islet cells in association with high leptin levels}

To further examine whether cell death may happen in in vitro model, we tested the expression of pro-apoptotic genes in pancreatic islets treated with leptin $(200 \mathrm{ng} / \mathrm{ml})$ or leptin plus high concentration of $\mathrm{AMH}(1 \mathrm{ng} / \mathrm{ml})$ for 2 hours was developed in a medium containing a high level of glucose. Our results showed that isolated islet cells exposure to high AMH $(1 \mathrm{ng} / \mathrm{ml})$ plus leptin levels (200 ng/ml) increased the expression levels of pro-apoptotic pro-

- Table 3 Serum metabolic parameters between PCOS women with normal and high AMH levels stratified by BMI.

\begin{tabular}{|c|c|c|c|c|c|c|}
\hline & \multicolumn{2}{|c|}{ Normal BMI (18.5-24.9 Kg/m²) } & \multirow[t]{2}{*}{ p-Value } & \multicolumn{2}{|c|}{ High BMI ( $\geq 25 \mathrm{Kg} / \mathrm{m}^{2}$ ) } & \multirow[t]{2}{*}{ p-Value } \\
\hline & Normal AMH & High AMH & & Normal AMH & High AMH & \\
\hline No. of subjects & 35 & 38 & & 22 & 19 & \\
\hline Age (years) & $26.94 \pm 4.38$ & $27.73 \pm 4.22$ & 0.229 & $26.95 \pm 5.54$ & $26.71 \pm 4.86$ & 0.442 \\
\hline Height (cm) & $159.50 \pm 5.22$ & $160.26 \pm 4.47$ & 0.274 & $159.43 \pm 5.12$ & $161.31 \pm 5.27$ & 0.141 \\
\hline Weight (kg) & $55.41 \pm 8.60$ & $55.12 \pm 6.28$ & 0.433 & $75.40 \pm 9.63$ & $74.03 \pm 10.17$ & 0.331 \\
\hline BMI $\left(\mathrm{kg} / \mathrm{m}^{2}\right)$ & $20.94 \pm 3.15$ & $21.20 \pm 2.20$ & 0.345 & $29.22 \pm 3.34$ & $28.42 \pm 2.80$ & 0.209 \\
\hline Serum AMH level (ng/ml) & $3.18 \pm 1.57$ & $10.95 \pm 4.69$ & $<0.001$ & $3.20 \pm 1.92$ & $14.48 \pm 8.75$ & $<0.001$ \\
\hline Basal LH level (mIU/ml) & $12.51 \pm 7.82$ & $12.05 \pm 7.25$ & 0.427 & $9.33 \pm 4.60$ & $8.21 \pm 4.71$ & 0.261 \\
\hline Basal FSH level (mIU/ml) & $6.07 \pm 1.51$ & $5.92 \pm 1.11$ & 0.375 & $5.66 \pm 1.44$ & $6.04 \pm 1.89$ & 0.266 \\
\hline Basal E2 level (pmol/l) & $180.22 \pm 93.14$ & $189.14 \pm 76.16$ & 0.381 & $170.65 \pm 100.26$ & $162.62 \pm 94.05$ & 0.414 \\
\hline TT level (nmol/I) & $2.01 \pm 0.87$ & $2.79 \pm 0.83$ & 0.003 & $1.96 \pm 0.81$ & $2.47 \pm 1.06$ & 0.700 \\
\hline Basal PRL level (ng/ml) & $14.83 \pm 8.57$ & $15.14 \pm 10.21$ & 0.456 & $14.97 \pm 7.56$ & $16.83 \pm 9.89$ & 0.283 \\
\hline Serum triglyceride (mM) & $1.77 \pm 0.99$ & $1.53 \pm 0.98$ & 0.174 & $1.86 \pm 1.16$ & $2.08 \pm 2.01$ & 0.331 \\
\hline Serum Cholesterol (mM) & $5.08 \pm 1.11$ & $4.72 \pm 1.57$ & 0.091 & $5.04 \pm 1.15$ & $5.17 \pm 1.04$ & 0.352 \\
\hline Serum HDL-c (mM) & $1.58 \pm 0.39$ & $1.41 \pm 0.35$ & 0.066 & $1.40 \pm 0.36$ & $1.20 \pm 0.35$ & 0.041 \\
\hline Serum LDL-c (mM) & $3.17 \pm 0.77$ & $2.61 \pm 0.94$ & 0.006 & $3.03 \pm 0.85$ & $3.16 \pm 1.02$ & 0.335 \\
\hline Fasting glucose (mM) & $4.94 \pm 0.66$ & $4.63 \pm 0.94$ & 0.059 & $4.99 \pm 0.83$ & $4.77 \pm 0.94$ & 0.211 \\
\hline Fasting insulin $(\mu \mathrm{U} / \mathrm{ml})$ & $10.71 \pm 4.98$ & $20.14 \pm 11.96$ & $<0.001$ & $11.98 \pm 6.95$ & $13.32 \pm 9.40$ & 0.302 \\
\hline HOMA-IR & $2.53 \pm 1.19$ & $2.46 \pm 1.95$ & 0.471 & $3.02 \pm 1.42$ & $3.96 \pm 1.27$ & 0.028 \\
\hline
\end{tabular}

Data = mean \pm SD; p-values were calculated by $t$-test. BMI: Body mass index; LH: Luteinizing hormone; FSH: Follicle-stimulating hormone; E2: Estradiol; TT: Total testosterone; PRL: Prolactin; HDL-c: High-density lipoprotein-cholesterol; LDL-c: Low-density lipoprotein-cholesterol; HOMA-IR: Homeostatic Model Assessment for Insulin Resistance. 
a

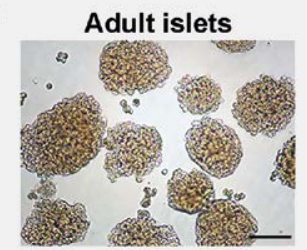

DTZ

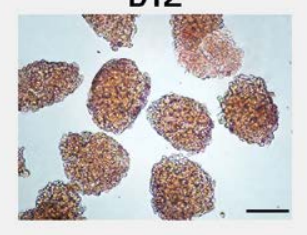

b

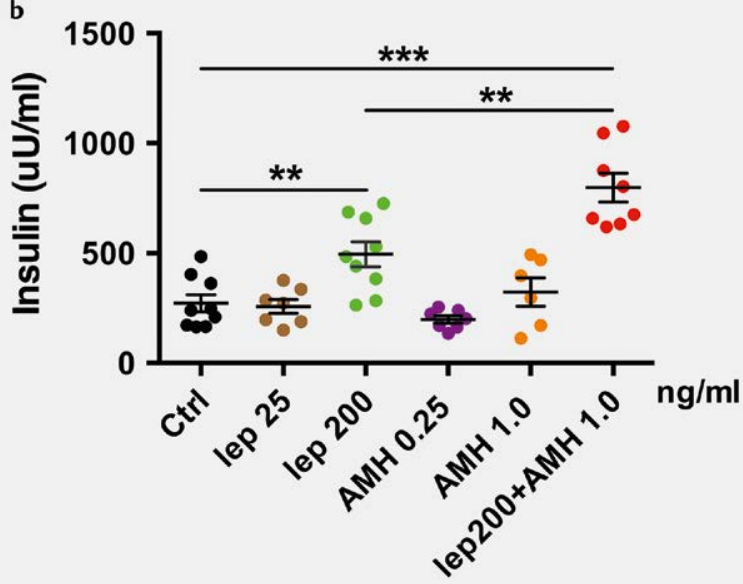

- Fig. 1 Co-treatment of high levels of AMH plus leptin increased insulin secretion. a: Adult islets were identified by incubating with dithizone (DTZ). Scale bar, $200 \mathrm{~mm}$. b: Insulin secretion in the groups treated with different concentration of leptin or AMH levels and high levels of $\mathrm{AMH}$ plus leptin.

teins, such as Bax, caspase-3, and caspase-8, compared to the group treated with leptin only ( $\vee$ Fig. $2 \mathbf{2 a - e}$ ).

\section{Discussion}

Our results illustrate that the possible average value for basal hormonal and lipids levels, HOMA-IR as well as insulin levels for both fasting and after glucose stimulation are significantly higher in PCOS patients compared to the controls. Multiple linear regression analysis showed that HOMA-IR was positively correlated to BMI and leptin levels, which indicated that PCOS women with high leptin level were susceptible to IR. Furthermore, elevated AMH levels were related to fasting insulin level and continuously insulin release after glucose administration, and, HOMA-IR was the highest in obese PCOS women with high AMH levels, which implied that high $\mathrm{AMH}$ might promote the intolerance of glucose and pathological process of IR.

IR is highly prevalent among PCOS patients, independent of obesity [18]. Hyperinsulinemia seems to be associated with hyperandrogenism, since insulin acts in the theca interna cells, potentiating the effects of luteinizing hormone (LH) on steroidogenesis and reducing hepatic production of sex-hormone-binding globulin, resulting in higher concentrations of free androgens [19]. In our daily clinical practice, AMH is widely used to observe ovarian response and oocyte yield. PCOS patients had significantly higher AMH levels compared to healthy fertile women [20]. Serum AMH level increased with antral follicle count (AFC), $\mathrm{LH}, \mathrm{T}$, and $\mathrm{E}_{2}$ in PCOS women and decreased with $\mathrm{BMI}$, age, and $\mathrm{FSH}$ in infertile control women [21]. Circulating $\mathrm{AMH}$ was well known to be play important role in ovarian follicle development and was considered to be a marker of reproductive ageing. Recent epidemiological investigation was surveyed in 3293 female participants and it was observed that lower age-specific AMH levels were associated with a higher risk of type 2 diabetes in women [22]. However, whether AMH is involved in part of the pathological process of IR remains unclear. It has been reported that there was a significant positive correlation between AMH levels and IR in non-obese adolescent females with PCOS [23]. But most studies have shown that women with PCOS and IR presented high AMH levels $[24,25]$. Hyperinsulinemia resulting from IR appears to increase the premature differentiation of the granulosa cells, suggesting that IR plays some role in AMH secretion by these cells. Caglar et al. [26] designed a study to evaluate the correlation between $\mathrm{AMH}$ levels and IR in normal-weight PCOS patients. Although the PCOS patients were not subdivided according to different phenotypes, no correlation was found between AMH levels and IR. Later on, Tian et al. [27] conducted a more comprehensive study involving more patients, and according to the results, no correlation was observed between $\mathrm{AMH}$ and indices of IR among all phenotypes of PCOS cases. In contrast, La Marca et al. [28] found a significant positive association between $\mathrm{AMH}$ and $\mathrm{IR}$, as well as an association between androgens and $\mathrm{AMH}$ in women with PCOS. Nevertheless, in relation to IR and PCOS, a direct correlation has been found between antral follicle count, ovarian volume and hyperinsulinemia $[24,29]$. So, it is reasonable to suggest that a high AMH level is a risk factor of IR. In our study, we found that women with high serum AMH levels positively correlated to fasting insulin and insulin levels of glucose tolerance, and the patients with high AMH levels presented higher insulin levels as well.

Considering that excess body fat is the primary predictor of insulin resistance in PCOS subjects [30], we then analyzed the insulin levels and HOMA-IR between PCOS women with normal or high AMH levels stratified by BMI. Indeed, women with $B M I \geq 25 \mathrm{Kg} / \mathrm{m}^{2}$ showed higher HOMA-IR then women with normal BMI. Importantly, PCOS women with $\mathrm{BMI} \geq 25 \mathrm{Kg} / \mathrm{m}^{2}$ and high $\mathrm{AMH}$ presented a higher HOMA-IR as well. But in PCOS women with normal BMI, women with high $\mathrm{AMH}$ showed an increased fasting insulin level, which indicated that $\mathrm{AMH}$ might promote the insulin secretion and played a role in the initial compensatory phase of diabetes, and once women underwent overweight or obesity, high AMH would play a very important role in the process of developing insulin resistance.

In addition, there are some indications that $\mathrm{AMH}$ may be directly involved in the physiology of target organs and increased the risk of disease [31,32]. In vitro studies have confirmed that elevated AMH can affect the primary-cultured human luteinizing granulosa cells to attenuate $\mathrm{FSH}$-induced estradiol and progesterone production [33]. High AMH level had an inhibitory effect on early human ovarian follicular development and suppressed the initiation of primordial follicle growth [34]. Taking these studies into account, we speculate that high peripheral AMH may exert an influence on pancreatic islets function and may increase the risk of IR and diabetes later in life, especially in obese PCOS women.

Using in vitro model, we demonstrated that r-leptin and $\mathrm{r}-\mathrm{AMH}$ exert effects on the primary islets. Isolated islet cells treatment with 
a

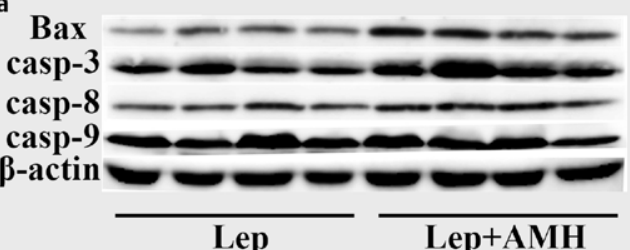

b

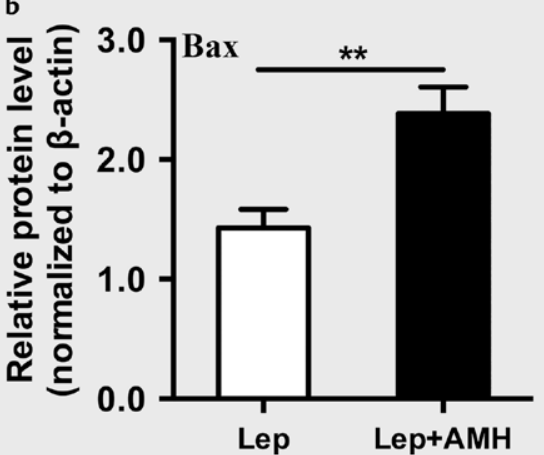

d

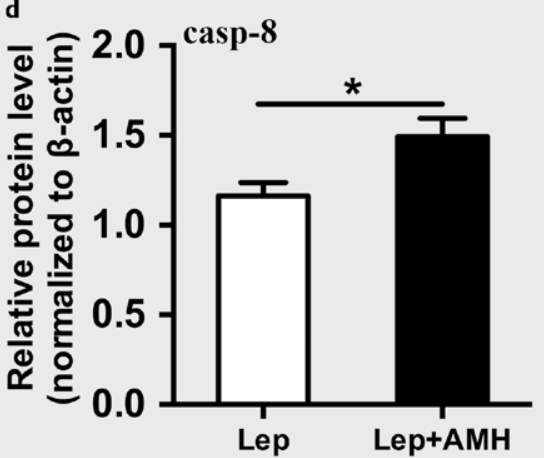

c
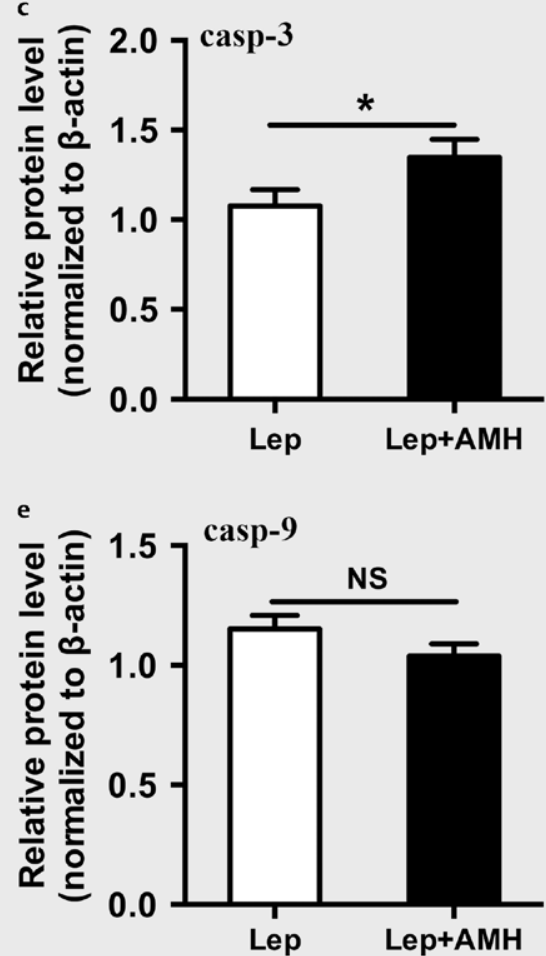

- Fig. 2 High AMH promotes apoptosis in pancreatic islet cells in association with high leptin levels. a: Protein expression of BAX, caspase-3, caspase- 8 and caspase- 9 in pancreatic islet cells treated with leptin ( $200 \mathrm{ng} / \mathrm{ml}$ ) or leptin plus high concentration of AMH ( $1 \mathrm{ng} / \mathrm{ml}$ ) for 2 hours ( $\mathrm{n}=4$, respectively). This figure shows one representative experiment among three separate experiments. $\mathbf{b}$ : Relative protein levels of BAX normalized to $\beta$-actin. c: Relative protein levels of caspase- 3 normalized to $\beta$-actin. d: Relative protein levels of caspase- 8 normalized to $\beta$-actin. e: Relative protein levels of caspase- 9 normalized to $\beta$-actin. The values are expressed as mean \pm SEM. ${ }^{*} p<0.05,{ }^{*}{ }^{*} p<0.01$.

leptin (200 ng/ml) or leptin plus AMH (1 ng/ml) significantly promoted insulin secretion. Interestingly, however, high levels of AMH non-significantly increase insulin secretion, compared to a group co-treated leptin plus AMH. Based on these results, we may suggest that high $\mathrm{AMH}$ in association with leptin might have a role in aggravating the impairment of pancreatic islet function. In order to overcome insulin resistance, the pancreatic islet $\beta$-cells releases more insulin initially to prevent hyperglycemia [35]. However, with the elevation of glucose continues to rise, this hyperactivity causes $\beta$-cell dysfunction that results in cell death [36]. For further analysis, we subjected the pancreatic islets to r-leptin or r-leptin plus $\mathrm{r}$-AMH to study the effect of high AMH on apoptosis of islets under the condition of high glucose. Caspases (cysteine-aspartic proteases, cysteine aspartases or cysteine-dependent aspartate-directed proteases) are a family of protease enzymes playing essential roles in programmed cell death (including apoptosis, pyroptosis, and necroptosis) and inflammation. Caspase- 3 is a converging point of the apoptotic pathway [37], and its peptide inhibitors have been shown to prevent islet apoptosis [38, 39]. Conversely, over-activation of some caspases such as caspase- 3 can lead to excessive programmed cell death.

As another pro-apoptotic protein, BAX plays a critical role in promoting apoptosis [40]. Bax can translocate from the cytosol to mitochondria in response to pro-apoptotic stimuli, resulting in mitochondrial cytochrome- $c$ release and activation of caspase- 9 and caspase-3 [41]. In addition, activated caspase- 8 can cleave Bid, which in turn triggers the release of cytochrome-c from mitochondria and the activation of caspase- 9 and caspase-3 [42]. Hyperglycemia-induced $\beta$-cell apoptosis has been implicated and has been studied mainly in $\mathrm{T}_{2} \mathrm{DM}$ [36]. We found that co-treatment of leptin plus AMH significantly increased the expression of Bax, caspase-3, and caspase- 8 in the pancreatic islets isolated from rats. 
The strength of our study is its prospective nature. All of the Rotterdam criteria were met for each PCOS diagnosis. To our knowledge, the study was designed for the first time to evaluate the association between $\mathrm{AMH}$ and IR in reproductive PCOS women. However, there are a few limitations that need to be addressed. First, the number of subjects was small in this study and thus do not wholly represent the general population. Second, more detail information, such as ovarian size and follicle count were not determined. Third, we only found co-treatment of high levels of AMH plus leptin increases insulin secretion and upregulated the expression of pro-apoptotic proteins when continuous incubating with high glucose level. The in vitro experiment is insufficient to state the relation of the pathogenesis of insulin resistance (IR) to high $\mathrm{AMH}$. Therefore, a well-designed case-control or cohort study needs to be performed and more experiments for investigating the relationship between $\mathrm{AMH}$ and apoptosis were needed in the future.

\section{Conclusions}

In obese PCOS patients, high levels of $\mathrm{AMH}$ may present an increased risk for IR. In vitro study showed that increasing AMH levels in pancreatic $\beta$-cells may trigger the secretion of insulin, and high $\mathrm{AMH}$ level in association with leptin promoted hyperglycemia-induced $\beta$-cell pro-apoptotic proteins expression. However, further study needs to fully clarify the molecular mechanism of $\mathrm{AMH}$-induced activation of apoptosis.

\section{Author Contributions}

X.H.L and H.F.H conceived, designed, and supervised the study as well as critically revising the manuscript; X.J.L and H.W collected the blood samples and wrote the manuscript; T.T.Y and K.U carried out the in vitro experiments; X.Y.S and H.Y.S did the hormone, lipids, and other blood tests; X.Y.F and Z.Y.L helped for the follow-up study and discussions.

\section{Funding Information}

This study was funded by the National Key Research and Development Program of China (No. 2018YFC1005001), the National Natural Science Foundation of China (No. 82071730) and Research Grant from Shanghai Hospital Development Center (No. SHDC12019X17).

\section{Conflict of Interest}

The authors declare that they have no conflict of interest.

\section{References}

[1] Dapas M, Lin FTJ, Nadkarni GN et al. Distinct subtypes of polycystic ovary syndrome with novel genetic associations: An unsupervised, phenotypic clustering analysis. PLoS Med 2020; 17: e1003132
[2] Rotterdam EA-SPcwg. Revised 2003 consensus on diagnostic criteria and long-term health risks related to polycystic ovary syndrome (PCOS). Hum Reprod 2004; 19: 41-47

[3] Dunaif A, Segal KR, Futterweit W et al. Profound peripheral insulin resistance, independent of obesity, in polycystic ovary syndrome. Diabetes 1989; 38: 1165-1174

[4] Weenen C, Laven JS, Von Bergh AR et al. Anti-Mullerian hormone expression pattern in the human ovary: Potential implications for initial and cyclic follicle recruitment. Mol Hum Reprod 2004; 10 : 77-83

[5] Pankhurst MW. A putative role for anti-Müllerian hormone (AMH) in optimising ovarian reserve expenditure. J Endocrinol 2017; 233: R1-R13

[6] Khan HL, Bhatti S, Suhail S et al. Antral follicle count (AFC) and serum anti-Müllerian hormone (AMH) are the predictors of natural fecundability have similar trends irrespective of fertility status and menstrual characteristics among fertile and infertile women below the age of 40 years. Reprod Biol Endocrinol 2019; 17: 20

[7] Dewailly D, Andersen CY, Balen A et al. The physiology and clinical utility of anti-Mullerian hormone in women. Hum Reprod Update 2014; 20: 370-385

[8] Visser JA, Themmen AP. Anti-Mullerian hormone and folliculogenesis. Mol Cell Endocrinol 2005; 234: 81-86

[9] Durlinger AL, Gruijters M], Kramer P et al. Anti-Mullerian hormone attenuates the effects of FSH on follicle development in the mouse ovary. Endocrinology 2001; 142: 4891-4899

[10] Allagnat F, Fukaya M, Nogueira TC et al. C/EBP homologous protein contributes to cytokine-induced pro-inflammatory responses and apoptosis in beta-cells. Cell Death Differ 2012; 19: 1836-1846

[11] Liu S, Du F, Li X et al. Effects and underlying mechanisms of irisin on the proliferation and apoptosis of pancreatic beta cells. PLoS One 2017; 12: e0175498

[12] Gillman MW. A life-course approach to obesity.In Kuh D, Ben-Shlomo Y, Eds A Life Course Approach to Chronic Disease Epidemiology. Oxford: Oxford University Press 2004: 189-217

[13] Matthews DR, Hosker JP, Rudenski AS et al. Homeostasis model assessment: Insulin resistance and beta-cell function from fasting plasma glucose and insulin concentrations in man. Diabetologia 1985; 28: $412-419$

[14] Martinez SC, Tanabe K, Cras-Meneur C et al. Inhibition of Foxo1 protects pancreatic islet beta-cells against fatty acid and endoplasmic reticulum stress-induced apoptosis. Diabetes 2008; 57: 846-859

[15] Ding GL, Wang FF, Shu J et al. Transgenerational glucose intolerance with Igf2/H19 epigenetic alterations in mouse islet induced by intrauterine hyperglycemia. Diabetes 2012; 61: 1133-1142

[16] He RH, Sheng JZ, Luo Q et al. Aquaporin-2 expression in human endometrium correlates with serum ovarian steroid hormones. Life Sci 2006; 79: 423-429

[17] Shanik MH, Xu Y, Skrha J et al. Insulin resistance and hyperinsulinemia: is hyperinsulinemia the cart or the horse? Diabetes Care 2008; 31 Suppl 2: S262-\$268

[18] Stepto NK, Cassar S, Joham AE et al. Women with polycystic ovary syndrome have intrinsic insulin resistance on euglycaemic-hyperinsulaemic clamp. Hum Reprod 2013; 28: 777-784

[19] Azziz R, Ehrmann DA, Legro RS et al. Troglitazone decreases adrenal androgen levels in women with polycystic ovary syndrome. Fertil Steril 2003; 79: 932-937

[20] Chao KC, Ho CH, Shyong WY et al. Anti-Mullerian hormone serum leve as a predictive marker of ovarian function in Taiwanese women. J Chin Med Assoc 2012; 75: 70-74

[21] Cui Y, Shi Y, Cui L et al. Age-specific serum antimullerian hormone levels in women with and without polycystic ovary syndrome. Fertil Steril 2014; 102: 230-236. , e232 
[22] Verdiesen RMG, Onland-Moret NC, van Gils CH et al. Anti-Müllerian hormone levels and risk of type 2 diabetes in women. Diabetologia 2021; 64: 375-384

[23] Tokmak A, Kokanali D, Timur $\mathrm{H}$ et al. Association between anti-Mullerian hormone and insulin resistance in non-obese adolescent females with polycystic ovary syndrome. Gynecol Endocrinol 2016; 32: 926-930

[24] Carmina E, Orio F, Palomba S et al. Ovarian size and blood flow in women with polycystic ovary syndrome and their correlations with endocrine parameters. Fertil Steril 2005; 84: 413-419

[25] Fonseca HP, Brondi RS, Piovesan FX et al. Anti-Mullerian hormone and insulin resistance in polycystic ovary syndrome. Gynecol Endocrinol 2014; 30: 667-670

[26] Caglar GS, Kahyaoglu I, Pabuccu R et al. Anti-Mullerian hormone and insulin resistance in classic phenotype lean PCOS. Arch Gynecol Obstet 2013; 288: 905-910

[27] Tian X, Ruan X, Mueck AO et al. Serum anti-Mullerian hormone and insulin resistance in the main phenotypes of non-obese polycystic ovarian syndrome women in China. Gynecol Endocrinol 2014; 30 : 836-839

[28] La Marca A, Orvieto R, Giulini S et al. Mullerian-inhibiting substance in women with polycystic ovary syndrome: Relationship with hormonal and metabolic characteristics. Fertil Steril 2004; 82: 970-972

[29] Pache TD, de Jong FH, Hop WC et al. Association between ovarian changes assessed by transvaginal sonography and clinical and endocrine signs of the polycystic ovary syndrome. Fertil Steril 1993; 59: 544-549

[30] Dahan MH, Reaven G. Relationship among obesity, insulin resistance, and hyperinsulinemia in the polycystic ovary syndrome. Endocrine 2019; 64: 685-689

[31] de Kat AC, Verschuren WM, Eijkemans MJ et al. Anti-mullerian hormone trajectories are associated with cardiovascular disease in women: Results from the doetinchem cohort study. Circulation 2017; 135: $556-565$
[32] McLennan IS, Pankhurst MW. Anti-Mullerian hormone is a gonadal cytokine with two circulating forms and cryptic actions. J Endocrinol 2015; 226: R45-R57

[33] Prapa E, Vasilaki A, Dafopoulos K et al. Effect of Anti-Mullerian hormone $(\mathrm{AMH})$ and bone morphogenetic protein 15 (BMP-15) on steroidogenesis in primary-cultured human luteinizing granulosa cells through Smad5 signalling. J Assist Reprod Genet 2015; 32: 1079-1088

[34] Carlsson IB, Scott JE, Visser JA et al. Anti-Mullerian hormone inhibits initiation of growth of human primordial ovarian follicles in vitro. Hum Reprod 2006; 21: 2223-2227

[35] Leahy JL. Pathogenesis of type 2 diabetes mellitus. Arch Med Res 2005; 36: 197-209

[36] Butler AE, Janson J, Bonner-Weir S et al. Beta-cell deficit and increased beta-cell apoptosis in humans with type 2 diabetes. Diabetes 2003; 52: $102-110$

[37] Emamaullee JA, Shapiro AM. Interventional strategies to prevent beta-cell apoptosis in islet transplantation. Diabetes 2006; 55: 1907-1914

[38] Nakano M, Matsumoto I, Sawada T et al. Caspase-3 inhibitor prevents apoptosis of human islets immediately after isolation and improves islet graft function. Pancreas 2004; 29: 104-109

[39] Cheng G, Zhu L, Mahato RI. Caspase-3 gene silencing for inhibiting apoptosis in insulinoma cells and human islets. Mol Pharm 2008; 5 : 1093-1102

[40] Scarfo L, Ghia P. Reprogramming cell death: BCL2 family inhibition in hematological malignancies. Immunol Lett 2013; 155: 36-39

[41] Yuan L, Li Y, Li G et al. Ang(1-7) treatment attenuates beta-cell dysfunction by improving pancreatic microcirculation in a rat model of Type 2 diabetes. J Endocrinol Invest 2013; 36: 931-937

[42] Gross A, Yin XM, Wang $\mathrm{K}$ et al. Caspase cleaved BID targets mitochondria and is required for cytochrome $c$ release, while $B C L-X L$ prevents this release but not tumor necrosis factor-R1/Fas death. J Biol Chem 1999; 274: 1156-1163 